\title{
PERFORMANCE ASSESSMENT OF A DIFFRACTION FIELD COMPUTATION METHOD BASED ON SOURCE MODEL
}

\author{
G.Bora Esmer ${ }^{1}$, Levent Onural ${ }^{1}$, Haldun M. Ozaktas ${ }^{1}$, Vladislav Uzunov ${ }^{2}$ and Atanas Gotchev ${ }^{2}$ \\ ${ }^{1}$ Bilkent University, Department of Electrical and Electronics Engineering, \\ TR-06800 Ankara, Turkey \\ ${ }^{2}$ Tampere University of Technology, Institute of Signal Processing, FIN-33101 Tampere, Finland
}

\begin{abstract}
Efficient computation of scalar optical diffraction field due to an object is an essential issue in holographic 3D television systems. The first step in the computation process is to construct an object. As a solution for this step, we assume that an object can be represented by a set of distributed data points over a space. The second step is to determine which algorithm provides better performance. The source model whose performance is investigated is based on superposition of the diffraction fields emanated from the hypothetical light sources located at the given sample points. Its performance is evaluated according to visual quality of the reconstructed field and its algorithmic complexity. Source model provides acceptable reconstructed patterns when the region in which the samples are given has a narrow depth along the longitudinal direction and a wide extent along the transversal directions. Also, the source model gives good results when the cumulative field at the location of each point due to all other sources tends to be independent of that location.
\end{abstract}

Index Terms - Diffraction, Optical Diffraction, Signal Reconstruction

\section{INTRODUCTION}

One of the fundamental problems within the scope of holographic 3DTV is the computation of the monochromatic scalar optical diffraction field from a given field elsewhere. Thus, performance of such a TV system will be affected by the performance of the utilized scalar optical diffraction calculation method. In the literature, there are several elegant ways to compute the scalar optical diffraction field, but most of them are based on some approximations [1-4]. Some of these are due to the employed diffraction models like Fresnel or Fraunhofer approximations. Some other approximations are due to the structure of object.

There are many methods in the literature that focus on the problem of diffraction field computation due to a set of

This work is supported by EC within FP6 under Grant 511568 with acronym 3DTV. distributed data points $[1-3,8-10]$. In some of these methods, each sample point is assumed as a point light source [1-3]. We call such methods as the "source model" based approaches. Although, the source model based approaches do not provide exact diffraction field under every scenario. They are widely used because of their ease in implementation. However, this way of the diffraction field computation may results in significant errors. Therefore, it is crucial to know when the source model can be used without introducing significant errors. This paper clarifies the conditions on the given data distribution over the space so that the source model gives acceptable results.

There is no doubt that when the computation is between two parallel planes, the source model gives exact results. However, the source model must be used with care for other cases.

There are some methods in the literature that provide to calculate the true diffraction field over the space [8-10]. We call these methods "field model".

\section{SIMULATIONS AND RESULTS}

The source model, which is based on superposition of the diffraction fields emanated from the object points, is widely used to calculate the diffraction field due to that object. However, having such an approach may result in a huge deviation from the exact solution because of coupling of the diffraction fields due to the sample points. In this section, to illustrate the amount of this deviation, some simulations which are based on different distribution of the given samples over the space are presented. Even if the source model is tested under many conditions, only a few of them can be given in this paper. Moreover, in this work, we deal with the diffraction fields from 1D signals over 2D space, but it can be extended to diffraction from 2D signals over 3D space as in [10].

In the presented scenarios, plane-wave decomposition (PWD), which is equivalent to Rayleigh-Sommerfeld (R-S) diffraction integral [5-7], is utilized to compute the diffraction field between two parallel lines. The discrete case of this relation can be expressed in matrix vector multiplication form 
as;

$$
\mathrm{g}=\mathbf{A f}
$$

where $\mathbf{f}$ and $\mathbf{g}$ are diffraction fields on the reference line and the other line which is parallel to the reference line, respectively. The matrix $\mathbf{A}$ can be decomposed as $\mathbf{A}=\mathbf{W}^{-1} \mathbf{H}_{p} \mathbf{W}$, where $\mathbf{W}$ is the $N$-point DFT matrix and $\mathbf{H}_{p}$ is a diagonal matrix,

$$
\mathbf{H}_{p}=\left[\begin{array}{cccc}
H_{p}(0) & 0 & \ldots & 0 \\
0 & H_{p}(1) & & 0 \\
\vdots & & \ddots & \vdots \\
0 & 0 & \ldots & H_{p}(N-1)
\end{array}\right]
$$

The diagonal elements, denoting the frequency response of the free space propagation, are calculated as

$$
H_{p}\left(n_{f}\right)=\exp \left[j \frac{2 \pi}{N}\left(\beta^{2}-n_{f}^{2}\right)^{\frac{1}{2}} p\right]
$$

where $\beta=\frac{N X_{s}}{\lambda}$ and $\lambda$ is the wavelength of the employed light. The parameter $X$ presents the spatial sampling period along transversal axis. The physical distance between two lines is expressed as $p X$. The sampling procedure, the details of the formulation and the underlying assumptions can be found in [8].

In the implemented scenarios, a simple function, which is a discrete unit-magnitude square pulse of 32 samples located at the center of a reference line, is used. Also, each transversal line in the space has $N=256$ samples and there are $M=N$ transversal lines. Sampling periods in both $x$ and $z$ axes are the same, and taken as $X=\lambda$.

Source model utilizes superposition of the diffraction fields due to the given data samples to obtain the diffraction field on the reference line. Diffraction field due to a sample point is expressed by a column vector, $\mathbf{r}\left(p_{i}, n_{i}\right)^{H}$ where $H$ denotes the conjugate transposition which is needed for backpropagation from the sample point to the reference line. This column vector is obtained from the $n_{i}^{t h}$ row vector of $\mathbf{A}$ matrix with $p=p_{i}$. Structure of the $\mathbf{A}$ matrix can be found in [8]. The source model can be summarized as

$$
\mathbf{f}=\sum_{i=1}^{s} y_{i} \mathbf{r}\left(p_{i}, n_{i}\right)^{H} .
$$

where $s$ is used to express the number of given samples over the space and $y_{i}$ denotes the source amplitude on $i^{\text {th }}$ given point. To determine the performance of the model, two criteria are taken into consideration. One of them is the algorithmic complexity and the other one is the visual quality of the reconstructed field on the reference line. We assume that computational complexity is dominated by the number of complex multiplications. To implement the source model, we need $s\left(\frac{N}{2} \log _{2} N+N\right)$ complex multiplications. The first term in the parenthesis comes from the inverse discrete Fourier transform (DFT) to compute $\mathbf{r}\left(p_{i}, n_{i}\right)$ for each point source. The second term is needed for the scaling of each column vector by the source amplitude. The expression within parenthesis has to be performed $s$ times to obtain the superposition over all given sample points.

Since we are using 1D simulations, there is no variation along one of the transversal directions when 2D optical masks are considered. Such a case is shown in Figure 1(a). The magnitude of the diffraction field, which is computed from the synthetically generated 1D diffraction field on the reference line, over the entire 2D space is shown in Figure 1(b).

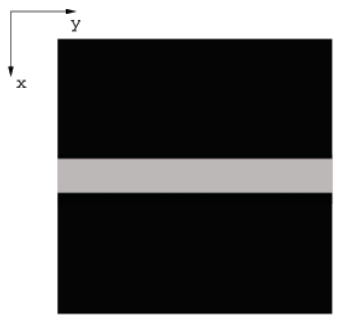

(a)

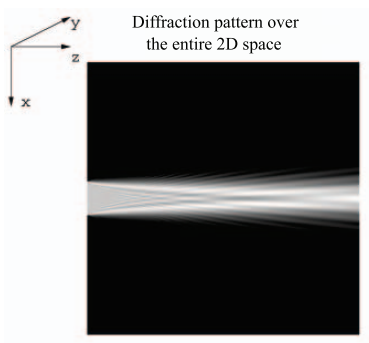

(b)
Fig. 1. (a) Magnitude of the synthetically generated diffraction field on the reference line. This is a 2D signal with 1D variation on it. (Images given in this paper has 256 grey levels)(b) Magnitude of the diffraction field over entire 2D space.

In the first set of implemented scenarios, the effect of the thickness of the region along the longitudinal axis is investigated. Therefore, the region in which the samples are given, is narrow along the longitudinal axis and has full extent along the transversal axis. Since our 1D array size is 256 in the implemented scenarios, we need at least 256 data points because the size of the 1D array determines the degrees of freedom of the problem. Assume that all the samples are given along the transversal direction then perfect reconstruction is possible. Therefore, we choose to take $1.2 * 256=307$ samples to overcome the bottleneck arises by the degrees of freedom of the problem. The locations of the sample points are shown in Figure 2. The magnitude of the reconstructed fields from the given samples are illustrated in Figure 3. Also, 1D plot of the reconstructed pattern, a transversal slice of the Figure 3(a), is shown in Figure 3(b) to give an insight to the reader about the shape of the reconstructed signal on the reference line by source model. As it can be seen from the Figure 3(a) and the Figure 3(f), there is a strip at the middle. However, when the depth of the region is increased gradually, the quality of the strip deteriorates as shown in Figures 3(c), 3(d) and 3(e).

In another scenario, the samples are taken along a diagonal line and the number of the given samples is decreased to 256, as illustrated in Figure 4. The distance between the consecutive sample points is set to $\sqrt{2} \lambda$. As it is seen from Figure 5 , the strip at the middle can be easily recognized. There is only a small amplitude variation on it. The reconstructed pattern is much better than the previous ones even if the num- 


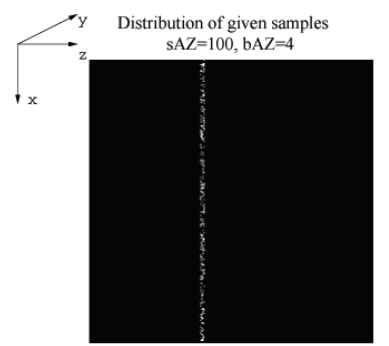

(a)

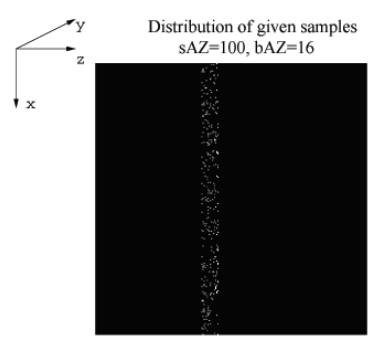

(c)

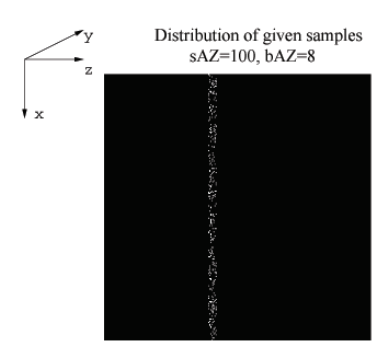

(b)

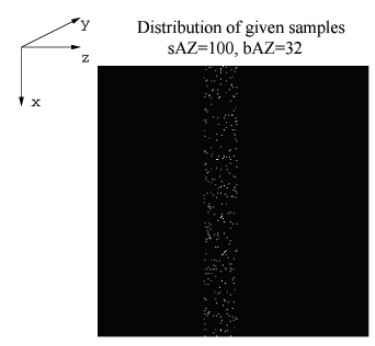

(d)

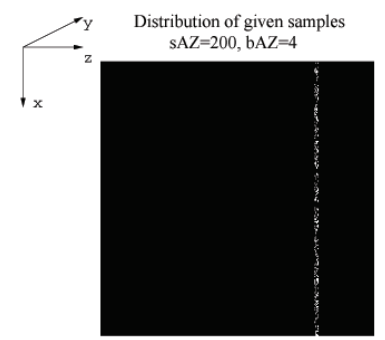

(e)

Fig. 2. (a) Locations of the given data points. The thickness of the volume which contains all data points is $4 \lambda$ and the distance between this volume and the reference line is $100 \lambda$. The number of given samples, $s$, is taken as 307 . (b) The thickness is $8 \lambda$. (c) The thickness is enlarged to $16 \lambda$. (d), to $32 \lambda$. (e) The depth of the region is kept as in Figure 2(a), but the distance is taken as $200 \lambda$.

ber of given samples is decreased to 256 .

Third scenario shows that we can obtain acceptable results by the source model when the given samples are accumulated within a tight region. In this scenario, the region is a circle whose radius is $30 \lambda$ and the number of given samples in it is 2822. The distribution of the samples is shown in Figure 6. The source model again works fine under this scenario. Magnitude of the reconstructed field can be seen in Figure 7.

\section{CONCLUSION}

This work presents an investigation of performance assessment of source model which is based on superposition of the diffraction fields emanated from the hypothetical light sources located at the given sample points under several scenarios. In

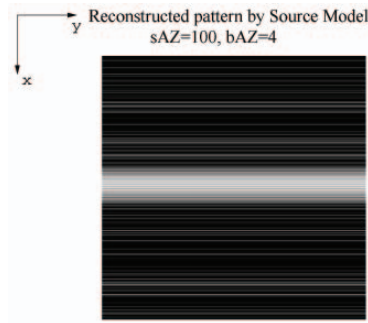

(a)



(c)

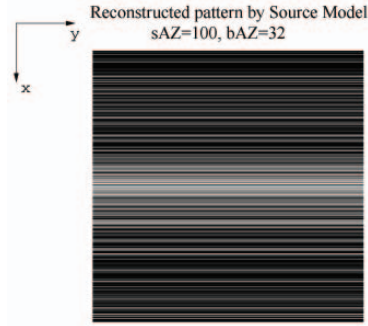

(e)

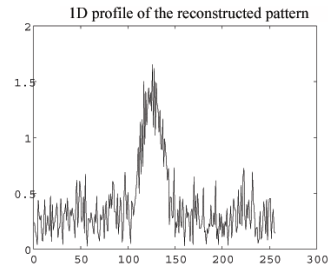

(b)

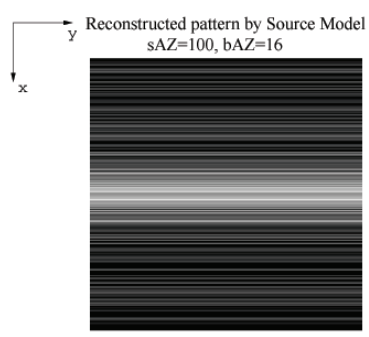

(d)

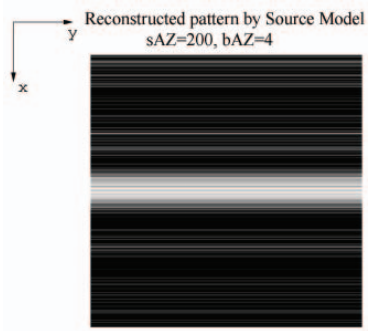

(f)
Fig. 3. (a) Magnitude of the reconstructed diffraction field on the reference line by the source model when the given data points are distributed as in Figure 2(a). (b) 1D profile of the same pattern in Figure 3(a). (c) Magnitude of the reconstructed field by the same method under the conditions given in Figure 2(b). (d) Obtained result when the given sample points are distributed as in Figure 2(c). (e) Reconstructed pattern when the samples are distributed as in Figure 2(d). (f) Magnitude of the reconstructed field on the reference line when the given samples as in Figure 2(e).

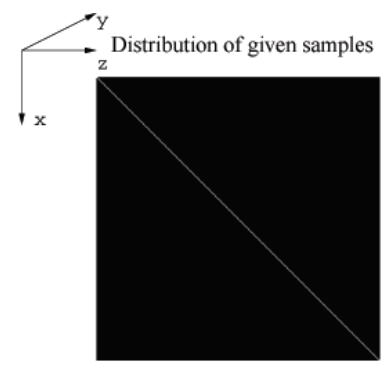

Fig. 4. Locations of the given samples in the second scenario.

these scenarios, the distribution of the given sample points over the space and their total number are changed. Evalua- 


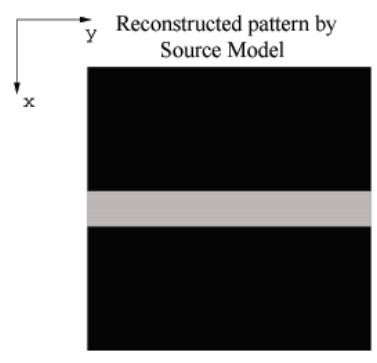

Fig. 5. Magnitude of the reconstructed diffraction field on the reference line by the source model. The given data points are distributed as in Figure 4.

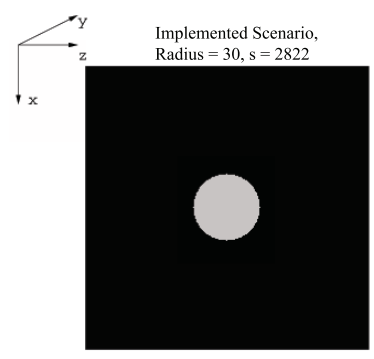

Fig. 6. Locations of the given samples in the third scenario. The radius of the circle is $30 \lambda$ and the distance between the center of the circle and the reference line is $128 \lambda$.

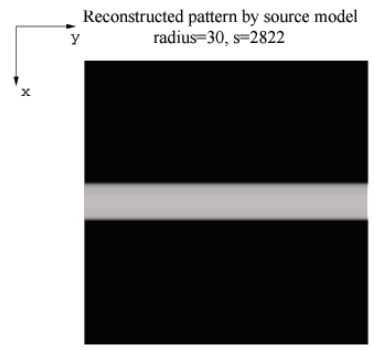

Fig. 7. Magnitude of the reconstructed field when the samples are distributed over the space as in Figure 6

tion of the performance is based on the visual quality of the reconstructed patterns and the algorithmic complexity of the method. The source model works fine when the cumulative field at the location of each point due to all other sources becomes negligible. Furthermore, even if the cumulative field due to all other sources is not small, there are cases where the source model yields quite good results. One such case is when the cumulative field tends to be independent of the location of each point.

\section{REFERENCES}

[1] M. Lucente, "Diffraction-specific fringe computation for electro-holography,”Doctral Thesis Dissertation,
MIT Dept. of Electrical Engineering, Dec. (1994).

[2] M. Matsushima, M. Takai, "Recurrence formulas for the fast creation of synthetic three-dimensional holograms", Appl. Optics, vol. 39, no. 35, 6587-6594, (2000).

[3] J. L. Juárez-Pérez, A. Olivares-Pérez, L. R. BerrielValdos, "Nonredundant calculations for creating digital Fresnel holograms", Appl. Optics, vol. 36, no. 29, 74377443, (1997).

[4] A. Ritter, J. Böttger, O. Deussen, M. König, T. Strothotte, "Hardware-based Rendering of Full-parallax Synthetic Holograms", Appl Optics, vol. 38, Issue 8, pp. 1364-1369, (1999).

[5] G. C. Sherman, "Application of the Convolution Theorem to Rayleigh's Integral Formulas," J. Opt. Soc. Am. 57, 546-547 (1967).

[6] É. Lalor, "Conditions for the Validity of the Angular Spectrum of Plane Waves," J. Opt. Soc. Am. 58, 12351237 (1968).

[7] L. Onural, "Exact Analysis of the Effects of Sampling of the Scalar Diffraction Field", J. Optical Soc. of America A, vol 24, no 2, pp 359-367, February (2007).

[8] G.B. Esmer, V. Uzunov, L. Onural, H.M. Ozaktas, A. Gotchev, "Diffraction field computation from arbitrarily distributed data points in space," Signal Processing: Image Communication, vol 22, no 2, pp 178-187, February (2007).

[9] V. Uzunov, A. Gotchev, G.B. Esmer, L. Onural, H. Ozaktas, "Non-Uniform Sampling and Reconstruction of Diffraction Field," TICSP Series 34, Florence, Italy (2006).

[10] G.B. Esmer, V. Uzunov, L. Onural, A. Gotchev and H.M. Ozaktas, "Reconstruction of Scalar Diffraction Field from Distributed Data Points Over 3D Space" 3DTV-CON 2007, Kos Island, Greece, 7-9 May (2007). 